\title{
Low-Energy Ionization Cooling of Ions for Beta Beam Sources
}

\author{
David Neuffer
}

Fermilab

PO Box 500, Batavia IL 60510

\begin{abstract}
Rubbia et al.[1] have recently suggested that multiturn passage of a low-energy ion beam (v/c $\cong$ 0.1) through a low-Z target can be used in the production of ions useable for beta-beam sources and that ionization cooling techniques can increase the circulating beam lifetime and thus enhance that production. Some parameters in their initial discussion are somewhat optimistic, and the conditions for 3-D cooling are not completely developed. In the present paper we reconsider some features of the scenarios and suggest some variations that may be more practical. While 3-D cooling is possible at these energies, mixing of longitudinal motion with both horizontal and vertical motion is necessary to obtain simultaneous cooling in all dimensions; we suggest lattice variations that would be needed. Direct and reverse kinematics are described and explored.
\end{abstract}

\section{Introduction}

An approach toward obtaining high-energy electron neutrino (and anti-neutrino) beams is the beta-beam scenario presented by Zucchelli.[2] In that scenario, accelerated beam is sent onto a target designed for the creation of unstable nuclei. The unstable nuclei are then collected and accelerated to moderately high energies and then transferred into a storage ring. Beta decay of the nuclei along the straight sections of the storage ring will provide electron neutrino beams, similar to the neutrino beams obtained from $\mu$-decay in $\nu$-factory scenarios.

The major difficulty is in obtaining enough neutrinos, and therefore enough appropriately unstable nuclei, and this requires a careful choice of initial beam, target, and product nucleus, with careful design of the entire collection system. The optimum product nucleus has a lifetime of $\sim 1 \mathrm{~s}$, since it must live long enough to be accelerated (with systems of cycle times of $\sim 1 \mathrm{~s}$ ) and must decay relatively quickly once stored. It is desirable that the decay energy (of the produced neutrinos) be as large as practical. Considerations of production and collection efficiency have set relatively low-Z nuclei as preferred cases, and Noble gases $(\mathrm{He}, \mathrm{Ne})$ are attractive since they will not bind chemically to the target material, and are relatively easily extracted. Complete exploration of the physics requires both an anti-neutrino (i. e., ${ }^{6} \mathrm{He}$ ) and a neutrino source $\left({ }^{18} \mathrm{Ne}\right)$, and neutrino sources are more difficult to obtain. Other nuclei are being considered with varying advantages and disadvantages.

In Rubbia et al.[1], the particular nuclei considered as antineutrino sources are ${ }^{8} \mathrm{Li}$ as the neutrino source and ${ }^{8} \mathrm{~B}$ as the neutrino source. These nuclei produce relatively high energy neutrinos. Rubbia proposes producing these by the reactions:

$$
{ }_{3}^{8} \mathrm{Li} \rightarrow{ }_{4}^{8} \mathrm{Be}+\mathrm{e}^{-}+\bar{v}_{\mathrm{e}}
$$




$$
{ }_{5}^{8} \mathrm{~B} \rightarrow{ }_{4}^{8} \mathrm{Be}+\mathrm{e}^{+}+v_{\mathrm{e}}
$$

The decay times for these are $0.84 \mathrm{~s}$ and $0.77 \mathrm{~s}$, respectively.

Ref. 1 suggests using a ${ }^{6} \mathrm{Li}$ or ${ }^{7} \mathrm{Li}$ beam as a circulating beam with multiturn passes through a ${ }^{2} \mathrm{H}$ or ${ }^{3} \mathrm{He}$ gas target to produce ${ }^{8} \mathrm{~B}$ or ${ }^{8} \mathrm{Li}$ following the reactions:

$$
\begin{aligned}
& { }_{3}^{6} \mathrm{Li}+{ }_{2}^{3} \mathrm{He} \rightarrow{ }_{5}^{8} \mathrm{~B}+\mathrm{n} \\
& { }_{3}^{7} \mathrm{Li}+{ }_{1}^{2} \mathrm{H} \rightarrow{ }_{3}^{8} \mathrm{Li}+\mathrm{p}
\end{aligned}
$$

The cross-section for these processes is $\sim 10 \mathrm{mb}$ for the B production and $\sim 100 \mathrm{mb}$ for the ${ }^{8} \mathrm{Li}$ production, out of total cross sections of $\sim 1000 \mathrm{mb}$. As with other beta-beam scenarios the neutrino source (B) is an order of magnitude more difficult than the antineutrino source ( $\mathrm{Li})$. The optimum energy for collisions is $\sim 20 \mathrm{MeV}$ (for Li ions on He target).

If a thick production target is used, the secondary ions will be trapped within the material. Therefore, ref. 1 proposes circulating the production beam many times through a thin production target, where it is relatively likely that produced radioactive ions can be extracted and accumulated before being reabsorbed. Reacceleration of the beam after every pass allows the primary beam to be reused for multiple production passes, keeping the beam at the same mean energy. The energy loss in the target and the reacceleration places the beam dynamics in a regime similar to that considered for ionization cooling of muons. In the present paper we explore this ionization cooling from the perspective of these previous ionization cooling studies, $[3,4]$ and compare that with the discussion of ref. 1.

A key difference is that energies used in the beta-beam source are nonrelativistic, with $\mathrm{v} / \mathrm{c} \cong 0.1$, while ionization cooling is more optimal at near-relativistic energies $(\mathrm{v} / \mathrm{c}>\sim 0.8)$. However ionization cooling of muons has been formulated in relativistically invariant coordinates, which apply for both cases.[3]

\section{Low Energy Ionization Cooling of Ions}

The rms ionization cooling equations for ions are, to an initial approximation, the same as those developed for muons, with the mass of the ion $\mathrm{m}_{\mathrm{I}}=\mathrm{am}_{\mathrm{N}}$ substituting for $\mathrm{m}_{\mu}$, and the charge $\mathrm{ze}$ substituting for the muon charge e where appropriate. At low energies, momentum $(\mathrm{P})$ is a more appropriate variable than energy for the discussion, where $\mathrm{P}$ is the ion momentum. In the following equations $\mathrm{z}$ and $\mathrm{a}$ are the incident ion nuclear charge and mass number, $\beta$ and $\gamma$ are the kinetic parameters of the incident ion. $\mathrm{Z}$ and $\mathrm{A}$ are the absorber material nuclear charge and mass. As first approximations for rms energy loss, multiple scattering and energy straggling for particles passing through material we have used the Bethe-Bloch equation and the Moliere equation and the Bohr formula[4], with the caveats that these formulae are not precisely accurate and may be somewhat different in the low energy dynamics of low-z nuclei scattering. These equations only include electromagnetic interactions of bare charges; nuclear scattering effects and recombination/ionization effects that are of importance for ions are not explicitly included in these first estimates.

The momentum loss coefficient is: 


$$
\frac{1}{\mathrm{P}} \frac{\mathrm{dP}}{\mathrm{ds}} \cong 4 \pi \mathrm{N}_{\mathrm{A}} \rho \mathrm{r}_{\mathrm{e}}{ }^{2} \mathrm{~m}_{\mathrm{e}} \mathrm{c}^{2} \frac{\mathrm{Z}}{\mathrm{A}} \frac{\mathrm{z}^{2}}{\beta^{2} \gamma \mathrm{am}_{\mathrm{N}} \mathrm{c}^{2}}\left[\frac{1}{\beta^{2}} \ln \left(\frac{2 \mathrm{~m}_{\mathrm{e}} \mathrm{c}^{2} \gamma^{2} \beta^{2}}{\mathrm{I}(\mathrm{Z})}\right)-1-\frac{\delta}{2 \beta^{2}}\right]
$$

where $\mathrm{am}_{\mathrm{N}}$ is the beam particle mass, $\mathrm{I}(\mathrm{Z})$ is the ionization energy of the target $\left(\mathrm{I}(\mathrm{Z}) \cong 16 \mathrm{Z}^{0.9}\right)$ and $\delta$ is the density effect correction. (In first approximations we have used $\delta=0$.)

The equation for transverse emittance cooling is :

$$
\frac{\mathrm{d} \varepsilon_{\mathrm{N}}}{\mathrm{ds}}=-\frac{1}{\mathrm{P}} \frac{\mathrm{dP}}{\mathrm{ds}} \varepsilon_{\mathrm{N}}+\frac{\beta \gamma \beta_{\perp}}{2} \frac{\mathrm{d}\left\langle\theta_{\mathrm{rms}}^{2}\right\rangle}{\mathrm{ds}}
$$

where $\beta_{\perp}$ is the tranverse focusing (betatron) function at the absorber, and the PDG expression for multiple scattering is:

$$
\frac{\mathrm{d}\left\langle\theta_{\mathrm{rms}}^{2}\right\rangle}{\mathrm{ds}}=\frac{\mathrm{z}^{2} \mathrm{E}_{\mathrm{s}}^{2}}{\beta^{2} \mathrm{c}^{2} \mathrm{P}_{\mathrm{a}}^{2} \mathrm{~L}_{\mathrm{R}}}\left(1+0.038 \ln \left(\mathrm{s} / \mathrm{L}_{\mathrm{R}}\right)\right)^{2},
$$

The $\ln \left(\mathrm{s} / \mathrm{L}_{\mathrm{R}}\right)$ expression shown here is a "small" correction effect that is not clearly defined in our example, and violates linearity (multiple scattering should be proportional to scattering length). There is an ambiguity in the multipass case whether $s$ should refer to the length of a single absorber or the integrated total length of absorber (or the length needed for one $\left(\mathrm{e}^{-1}\right)$ cooling period). In muon cooling we have initially ignored this correction in first approximation, and obtain good agreement with simulations. That may be pessimistic in the present examples. (With $\mathrm{s}=0.018 \mathrm{~cm}, \mathrm{~L}_{\mathrm{R}}=756 \mathrm{~cm}(\sim \mathrm{He}$ liquid $), \mathrm{K}_{\mathrm{s}}=\left(1+0.038 \ln \left(\mathrm{s} / \mathrm{L}_{\mathrm{R}}\right)\right)=0.6$. $)$ In the present paper, we substitute a correction factor $\mathrm{K}_{\mathrm{s}}$ for $\left(1+0.038 \ln \left(\mathrm{s} / \mathrm{L}_{\mathrm{R}}\right)\right)$ and note possible variations.

Lynch and Dahl [5] have proposed a formula for $\mathrm{K}_{\mathrm{s}}$ that, from their analysis of Moliere scattering, is more accurate for low- $\beta$ beams. That expression is:

$$
\mathrm{K}_{\mathrm{s}}=\left[1+0.038 \ln \left(\frac{\mathrm{sz}}{\mathrm{L}_{\mathrm{R}} \beta^{2}}\right)\right]
$$

For these example values $(\mathrm{s}=0.018 \mathrm{~cm}, \mathrm{z}=3, \beta=0.1), \mathrm{K}_{\mathrm{s}}=0.86$ and the correction factor is much closer to 1 .

The resulting cooling equation is:

$$
\frac{\mathrm{d} \varepsilon_{\mathrm{N}}}{\mathrm{ds}}=-\frac{J_{\mathrm{t}}}{\mathrm{P}_{\mathrm{a}}} \frac{\mathrm{dP}_{\mathrm{a}}}{\mathrm{ds}} \varepsilon_{\mathrm{N}}+\frac{\beta_{\perp} \mathrm{z}^{2} \mathrm{E}_{\mathrm{s}}^{2} K_{\mathrm{s}}^{2}}{2 \beta^{2} \mathrm{am}_{\mathrm{N}} \mathrm{c}^{3} \mathrm{~L}_{\mathrm{R}} \mathrm{P}_{\mathrm{a}}}
$$

where $\varepsilon_{N}$ is the transverse normalized emittance, $J_{t}$ is the transverse partition number $\left(J_{x}=J_{y}=1\right.$ without emittance exchange), $\beta=\mathrm{v} / \mathrm{c}, \mathrm{P}_{\mathrm{a}}$ is the ion momentum, $\mathrm{dP}_{\mathrm{a}} / \mathrm{ds}$ is the momentum loss in the absorber, $E_{s}$ is the characteristic scattering energy $(\sim 14 \mathrm{MeV}), L_{R}$ is the characteristic radiation length in the material, and $\beta \perp$ is the transverse focusing function at the absorber. We have added the reference to the partition number as it will become non-unity when transverse and longitudinal coolings are mixed. 
The major difficulty in lower energy cooling is that the increase of energy loss with reduced energy heats the beam longitudinally, increasing the longitudinal emittance. The equation for rms energy spread change is:

$$
\frac{\mathrm{d} \sigma_{\mathrm{E}}^{2}}{\mathrm{ds}}=-2 \frac{\partial \frac{\mathrm{dE}}{\mathrm{ds}}}{\partial \mathrm{E}} \sigma_{\mathrm{E}}^{2}+\frac{\mathrm{d}\left\langle\Delta \mathrm{E}_{\mathrm{rms}}^{2}\right\rangle}{\mathrm{ds}},
$$

where the second is the heating term caused by random fluctuations in the particle energy loss. In the long-pathlength Gaussian-distribution limit, the second term in Eq. 2 is approximated by:

$$
\frac{\mathrm{d}\left\langle\Delta \mathrm{E}_{\mathrm{rms}}{ }^{2}\right\rangle}{\mathrm{ds}}=4 \pi\left(\mathrm{r}_{\mathrm{e}} \mathrm{m}_{\mathrm{e}} \mathrm{c}^{2}\right)^{2} \mathrm{n}_{\mathrm{e}} \mathrm{z}^{2} \gamma^{2}\left(1-\frac{\beta^{2}}{2}\right)
$$

The longitudinal cooling equation (eq. 5) only tracks energy spread. It can be transformed into a longitudinal emittance cooling equation, by adding longitudinal $\mathrm{rf}$ focusing that places the beam within a bunch. We also change variables to $\mathrm{z}, \delta$, where $\delta=\delta \mathrm{P} / \mathrm{P}$ to more closely follow the notation used for transverse motion $(\mathrm{x}, \theta)$ :

$$
\frac{\mathrm{d} \varepsilon_{\mathrm{z}}}{\mathrm{ds}}=-\frac{\mathrm{J}_{\mathrm{z}}}{\mathrm{P}} \frac{\mathrm{dP}}{\mathrm{ds}} \varepsilon_{\mathrm{z}}+\frac{\beta_{\mathrm{L}} \beta \gamma}{2} \frac{\mathrm{d}\left\langle\delta_{\mathrm{rms}}{ }^{2}\right\rangle}{\mathrm{ds}}
$$

Here $\varepsilon_{z}$ is a normalized longitudinal emittance. The heating or cooling effect of energy-loss is expressed as a partition number $\mathrm{J}_{\mathrm{z}}$ where the partition number is the relative rate of cooling or heating compared to the fractional momentum change:

$$
J_{z}=\frac{\frac{d \varepsilon_{L} / d s}{\varepsilon_{L}}}{\frac{d p / d s}{p}}=\frac{\frac{\partial(d E / d s)}{\partial E}}{\frac{d p / d s}{p}}
$$

(Ref. 3 uses $g_{i}$ rather than $J_{i}$ for partition numbers; we use $J_{i}$ for notation consistency with radiation damping.)

From the Bethe-Bloch expression for $\mathrm{dE} / \mathrm{ds}$ (with $\delta=0$ ), this is:

$$
J_{\mathrm{z}} \cong-\frac{2}{\gamma^{2}}+\frac{2\left(1-\frac{\beta^{2}}{\gamma^{2}}\right)}{\left(\ln \left[\frac{2 \mathrm{~m}_{\mathrm{c}}{ }^{2} \beta^{2} \gamma^{2}}{\mathrm{I}(\mathrm{z})}\right]-\beta^{2}\right)}
$$

(This is $\sim-1.6$ at $\mathrm{v} / \mathrm{c} \cong 0.1$ ). The transverse ( $\mathrm{x}$ and $\mathrm{y}$ ) partition numbers are both 1 (without emittance exchange). So, at the energies considered for beta-beam source cooling, $\Sigma \mathrm{J}_{\mathrm{i}} \cong 0.4$, and potential 6-D cooling is relatively weak.

$\beta_{L}$ is a focusing function, determined by the longitudinal focusing:

$$
\beta_{\mathrm{L}}=\sqrt{\frac{\left\langle\mathrm{z}^{2}\right\rangle}{\left\langle\delta^{2}\right\rangle}}=\sqrt{\frac{\beta^{2} \mathrm{PcC} \lambda_{\mathrm{RF}}\left(\frac{1}{\gamma^{2}}-\frac{1}{\gamma_{\mathrm{T}}^{2}}\right)}{2 \pi \mathrm{zeV} \mathrm{VF}_{\mathrm{RF}} \sin \varphi_{\mathrm{S}}}}
$$

where $\lambda_{R F}$ is the $\mathrm{rf}$ wavelength, $e V_{R F} \sin \phi_{s}$ is the mean focusing $\mathrm{rf}$ voltage, $\mathrm{C}$ is the ring circumference, and $\alpha_{p}$ is the momentum compaction. $\left(\alpha_{p}=1 / \gamma^{2}-1 / \gamma_{t}^{2}\right)$. In $\delta$ units the energy straggling term is: 


$$
\frac{\mathrm{d}\left(\delta_{\mathrm{rms}}{ }^{2}\right)}{\mathrm{ds}}=4 \pi\left(\mathrm{r}_{\mathrm{e}} \mathrm{m}_{\mathrm{e}} \mathrm{c}^{2}\right)^{2} \mathrm{~N}_{\mathrm{A}} \frac{\mathrm{Z}}{\mathrm{A}} \rho \frac{\gamma^{2} \mathrm{z}^{2}}{\beta^{2} \mathrm{P}_{\mathrm{a}}^{2}}\left(1-\frac{\beta^{2}}{2}\right)
$$

We insert a caveat here that this rms straggling equation and the above scattering term are only first approximations, and actual results may differ, particularly at small v/c.

As discussed previously, the cooling derivative $\partial(\mathrm{dE} / \mathrm{ds}) / \partial \mathrm{E}$ can be changed by placing the beam in a dispersive region $(x \rightarrow x+\eta \delta)$, where $\eta$ is the dispersion, and using a wedge absorber $\left(\rho^{\prime} / \rho_{o}\right.$ gives the change of density with respect to $\mathrm{x}$ ). The result is that:

$$
\frac{\partial\left(\frac{\mathrm{dE}}{\mathrm{ds}}\right)}{\partial \mathrm{E}} \rightarrow \frac{\partial\left(\frac{\mathrm{dE}}{\mathrm{ds}}\right)}{\partial \mathrm{E}}+\frac{\mathrm{dE}}{\mathrm{ds}} \frac{\eta \rho^{\prime}}{\beta \rho_{0} \mathrm{cP}}
$$

The wedge changes the partition number $J_{z}$ by $\eta \rho^{\prime} / \rho_{0}$. This couples with the transverse motion, and reduces the transverse cooling by the same degree $\left(J_{x} \rightarrow\left(1-\eta \rho^{\prime} / \rho_{0}\right)\right)$, so that the sum of cooling rates remains the same. The dispersion coupling also mixes the heating terms, adding a term of $\beta \gamma \eta^{2} / 2 \beta_{\mathrm{x}} \mathrm{d} \delta^{2} / \mathrm{ds}$ to the transverse emittance growth and $\beta \gamma \eta^{2} / 2 \beta_{\mathrm{L}} \mathrm{d} \theta^{2} / \mathrm{ds}$ to the longitudinal emittance growth[6]. These heating effects are "small" in "well-designed" systems; however, that must be checked for beta-beam source cooling. (For example, if $\beta_{\mathrm{x}}=0.3 \mathrm{~m}$, $\eta=0.3 \mathrm{~m}$ and $\beta_{\mathrm{L}}=6 \mathrm{~m}$ the coupling terms are $\sim 5 \%$ of the direct terms for $25 \mathrm{MeV}{ }^{6} \mathrm{Li}$ cooling by ${ }^{3} \mathrm{He}$.)

\section{Comments on Rubbia et al. [1]}

In this section we discuss some features of Ref. 1 and develop some of its discussion to more direct comparisons with previous beam cooling studies.

Ref. 1 is inconsistent in its discussion of cooling between transverse and longitudinal cooling. In the transverse dimensions ( $\mathrm{x}$ and $\mathrm{y}$ ), the cooling of the transverse emittances is calculated and the results can be directly compared and correlated with previous discussions of beam cooling, including radiation damping and previous ionization cooling papers.

However in the longitudinal direction the discussion is presented in terms of energy spread, and the longitudinal emittance growth or cooling is not directly addressed. Thus the correlation and coupling with transverse emittance growth or cooling is not completely addressed, and the conditions for cooling in three dimensions (6-D phase space) are not properly developed.

Another difficulty, which is more readily corrected, is the choice of (kinetic) energy rather than momentum as the reference quantity in developing the cooling discussion. The difficulty in use of energy is that at low energies non-relativistic kinetic energy is the more natural unit and at high energies relativistic total energy is more natural. With momentum, the relativistic momentum is useable at all energies and the transition from relativistic to nonrelativistic cooling is natural. (For example, the cooling partition numbers are naturally developed in momentum.) Also, the position change due to dispersion is defined in terms of $\delta \mathrm{p} / \mathrm{p}: \quad \delta \mathrm{x}=\mathrm{D}_{\mathrm{x}} \delta \mathrm{p} / \mathrm{p}$. However, for the present discussion, one can simply use the nonrelativistic conversion to switch between units: $\delta \mathrm{p} / \mathrm{p}=\delta \mathrm{T} /(2 \mathrm{~T})=\delta \mathrm{E} /(2 \mathrm{~T})$, where $\mathrm{T}$ is kinetic energy, and $\mathrm{E}$ is total energy. 
In the discussion of energy cooling (section 5 of ref. 1), it is noted that synchrotron oscillations mix phase and energy motions with the result that the energy offset varies as:

$$
\Delta E=A e^{-\alpha_{e} t} \cos \left(\Omega_{s} t\right), \text { where } \alpha_{e}=\frac{1}{2 P_{S}}\left[\frac{d U}{d E}\right],
$$

Where $P_{S}$ is the revolution period and $\mathrm{dU} / \mathrm{dE}$ is the variation of energy loss with respect to particle energy and $\alpha_{e}$ is the energy width damping coefficient. However, we note that the phase width has the same damping coefficient, and the product of these, which is the longitudinal emittance, has a damping coefficient of $\alpha_{\mathrm{z}}=2 \alpha_{\mathrm{e}}$. This longitudinal emittance damping coefficient is the quantity that should be compared with the transverse emittance coefficients; not the energy coefficient.

Ref. 1 discusses the particular case of the cooling of $25 \mathrm{MeV}{ }^{6} \mathrm{Li}$ ions with $\mathrm{U}=0.3 \mathrm{MeV}$ energy loss per turn (in ${ }^{3} \mathrm{He}$ ), and notes that $\mathrm{dU} / \mathrm{dE}$ is -0.0096 which corresponds to antidamping. This means a 1/e growth time of 208 turns in energy spread, or, for comparison with transverse emittance damping, of 104 turns in emittance.

The dependence of energy loss with energy can be changed by using a wedge absorber at a position with nonzero dispersion $D_{x}$, and the effect of the wedge absorber (whether using $\alpha_{z}$ or $\left.\alpha_{e}\right)$ is that $U \rightarrow U+U^{\prime} D_{x} \delta p / p=U+U^{\prime} D_{x} \delta E /(2 T)$, where $U^{\prime}$ is the derivative of energy loss with respect to position caused by the wedge. This means $\mathrm{dU} / \mathrm{dE}$ is replaced by:

$$
\left.\frac{d U}{d E} \rightarrow \frac{d U}{d E}\right|_{0}+\frac{U^{\prime} D_{x}}{2 T}
$$

Thus to obtain damping in $\alpha_{\mathrm{e}}$ or $\alpha_{\mathrm{z}}$ in the example, we must have $\mathrm{U}^{\prime} \mathrm{D}_{\mathrm{x}}>2 \mathrm{~T} \mathrm{dU} / \mathrm{dE}$ or $0.48 \mathrm{MeV}$.

The difficulty with this is revealed in equation (24) of ref. 1, which is correctly derived. The damping coefficient for horizontal emittances is changed by the wedge to:

$$
\alpha_{X}=\frac{1}{2 T P_{S}}\left(U_{0}-U^{\prime} D_{x}\right)
$$

This threshold value of $U^{\prime} D_{x}$ for longitudinal cooling is greater than $\mathrm{U}_{0}=0.3 \mathrm{MeV}$, and therefore any value that changes the motion from longitudinal heating to longitudinal damping also changes the motion to horizontal emittance antidamping.

The situation is clarified by rewriting the expression for longitudinal emittance damping:

$$
\alpha_{z}=\frac{1}{P_{S}}\left[\left.\frac{d U}{d E}\right|_{0}+\frac{U^{\prime} D_{x}}{2 T}\right]
$$

and recognizing that the change in emittance damping in longitudinal motion from the wedge is exactly opposite to that in horizontal, but the sum of damping rates $\alpha_{x}+\alpha_{z}$ is constant. This invariant has been previously recognized in ionization cooling (since 1982) and in radiation damping. The situation is further clarified by interpreting the comment "the damping time for vertical ... is that for energy losses twice the kinetic energy" to momentum units: the damping time is the time for momentum loss equal to the particle momentum, which implies partition 
numbers $\mathrm{J}_{\mathrm{y}}=1$ and $\mathrm{J}_{\mathrm{x}}=1$ (without wedge), just as in previous ionization cooling analyses. The longitudinal partition number (without wedge) at this low energy is

$$
J_{Z}=\left.\frac{2 T}{U_{0}} \frac{d U}{d E}\right|_{0}=-1.6 \text {, }
$$

which is in close agreement with the previous analyses of ionization cooling of muons, extrapolated to ions. (see fig. 1) Under wedge perturbation, damping is exchanged among $\mathrm{x}, \mathrm{y}$, and $\mathrm{z}$ but $\Sigma \mathrm{J}_{\mathrm{i}}$ is constant. The analyses indicate that you cannot obtain cooling in all 3 dimensions (6-D phase space) without mixing both $x$ and $y$ with $z$. Ref. 1 limits exchange to between $\mathrm{x}$ and $\mathrm{z}$.

Comparisons with ref. 1 present several difficulties. Section 6 of ref. 1 presents an example value of $U^{\prime} D_{x}=2 U_{0}$, which would damp longitudinally but heat the beam horizontally, and the text states that cooling horizontally occurs at a rate of half of $\left(\mathrm{U}_{\mathrm{o}} / 2 \mathrm{TP}_{\mathrm{s}}\right)$, which would disagree with eq. (24) of ref. 1. Section 7 of ref. 1 then presents "simulation results" that appear to show cooling simultaneously in $\mathrm{x}, \mathrm{y}$, and $\delta \mathrm{E}$. However, the parameters ignore longitudinal motion $(\phi$ or $\delta \mathrm{z}$ ) and may not include synchrotron motion. 6-D simulations with explicit longitudinal emittances will be needed to resolve the discrepancy. (The results would appear to be consistent with using $U^{\prime} D_{x}=2 U_{0}$ for the longitudinal motion simulation and $U^{\prime} D_{x}=U_{0} / 2$ for the horizontal motion simulation.)

However, at the given parameters, $\Sigma \mathrm{J}_{\mathrm{i}} \cong 0.4$ and is damping and it is in principle possible to have damping in $\mathrm{x}, \mathrm{y}$, and $\mathrm{z}$ by mixing longitudinal cooling with both vertical and horizontal. With equal cooling rates $\mathrm{J}_{\mathrm{i}} \cong 0.13$, however, and the equilibrium emittances and cooling times are then somewhat larger than those discussed in ref. 1. (For comparison, $\Sigma \mathrm{J}_{\mathrm{i}} \cong 4$ for radiation damping, and $\Sigma \mathrm{J}_{\mathrm{i}} \cong 2$ for relativistic ionization cooling.)

\section{Cooling and parameters for beta-beam examples}

In this section we develop the parameters for beta-beams with ionization cooling. We begin with parameters similar to those developed in ref. 1, but adapted by the discussion above. For a first example we consider Li-6 beam with a He-3 absorber. The Li-6 kinetic energy is $25 \mathrm{MeV}$, which implies a momentum $P_{a}=530 \mathrm{MeV} / \mathrm{c}$, and $\beta=0.094$. The ions are assumed to be stored within the ring until a nuclear interaction occurs. In ref. 1 the total cross section for Li-6 He-3 collisions is estimated at 1 barn $\left(10^{-24} \mathrm{~cm}^{2}\right)$ with only 0.01 barn producing the desired B-8 ion. To obtain $1 \mathrm{rms}$ interaction, a Li ion must pass through $\sim 5 \mathrm{gm} / \mathrm{cm}^{2}$ of He-3. The reference cooling length in ionization cooling is $(\mathrm{dP} / \mathrm{ds} / \mathrm{P})^{-1}$, which is $0.45 \mathrm{~cm}$ at liquid He-3 density or $0.042 \mathrm{gm} / \mathrm{cm}^{2}$. This is $\sim 1 / 100$ of the nuclear interaction length, which indicates that the beam would blow-up longitudinally in that period, unless cooled by balancing the $\mathrm{x}, \mathrm{y}, \mathrm{z}$ cooling rates.

According to the above discussion the present example has an efficiency of, at best, 0.01 B- 8 ion per Li-6 ion. To accumulate $10^{13}{ }^{8} \mathrm{~B}$ ions per second we need at least $10^{15}{ }^{6} \mathrm{Li}$ ions/s. Ref. 1 assumes that space charge limits the ${ }^{6} \mathrm{Li}$ beam storage to $\sim 10^{12}$ ions at a time, which would imply that the ring must be refilled every ms. If the ring circumference is $\mathrm{C}=10 \mathrm{~m}$, we then find $\sim 3000$ turns of storage are needed per fill, with an absorber thickness of $\sim 1.67 \times 10^{-3} \mathrm{gm} / \mathrm{cm}^{2}$ (or $0.018 \mathrm{~cm}$ at liquid density) to obtain a 1 barn interaction length. This is an energy loss of $1.87 \mathrm{MeV} /$ turn. The cooling time is 26.7 turns at $\mathrm{J}_{\mathrm{i}}=1$. 
As we discuss above, coupling among both transverse dimensions and the longitudinal dimension is necessary for 3-D (6-D phase-space) cooling. It is not too difficult to obtain a lattice with that property. As a simplest example we consider a lattice with a single solenoid within the ring with its strength set to exchange $\mathrm{x}$ and $\mathrm{y}$ phase space in a single turn, a variation of the "Moebius accelerator" concept.[7] This can occur if the integrated field BL of the solenoid is equal to $\pi \mathrm{B} \rho$, where $\mathrm{B} \rho$ is $\mathrm{p} / \mathrm{ze}=0.589 \mathrm{~T}-\mathrm{m}$, implying $\mathrm{BL}=1.85 \mathrm{~T}-\mathrm{m}$. With that exchange, a horizontal wedge would mix $\mathrm{x}$ and $\mathrm{z}$ and $\mathrm{y}$ with $\mathrm{z}$ on alternate turns, and a balanced exchange is possible that could have $\mathrm{J}_{\mathrm{x}}=\mathrm{J}_{\mathrm{y}}=\mathrm{J}_{\mathrm{z}} \cong 0.13$. At $\mathrm{J}=0.13$, the cooling time is $\sim 205$ turns.

With these parameters, and values of $\beta_{x}=0.3 \mathrm{~m}$ and $\eta=0.3 \mathrm{~m}$ at the absorber, we find equilibrium transverse emittances as approximated by:

$$
\varepsilon_{\mathrm{N}, \mathrm{eq}}=\frac{\beta_{\perp} \mathrm{z}^{2} \mathrm{E}_{\mathrm{s}}^{2} K_{s}^{2}}{2 J_{t} \beta \mathrm{am}_{\mathrm{N}} \mathrm{c}^{2} \mathrm{~L}_{\mathrm{R}} \frac{d E}{d s}}
$$

At the reference parameters, $\varepsilon_{\mathrm{N}, \text { eq }} \cong 4.35 \times 10^{-5} \mathrm{~K}_{\mathrm{s}}{ }^{2}$. The rms beam size is:

$$
\sigma_{\mathrm{X}}(\mathrm{s})=\mathrm{K}_{\mathrm{s}} \sqrt{\frac{\beta_{\perp}(\mathrm{s}) \varepsilon_{\mathrm{N}}}{\beta \gamma}},
$$

which is $1.5 \mathrm{~cm}$ at $\beta_{\mathrm{x}}=1 \mathrm{~m}, \mathrm{~K}_{\mathrm{s}}=0.7$. The rms scattering angle is

$$
\theta_{\mathrm{x}}(\mathrm{s})=\mathrm{K}_{\mathrm{s}} \sqrt{\frac{\varepsilon_{\mathrm{N}}}{\beta_{\perp}(\mathrm{s}) \beta \gamma}} \text {, which is } 0.0275 \text { at } \beta_{\mathrm{x}}=0.3 \mathrm{~m}, \mathrm{~K}_{\mathrm{s}}=0.7 \text {. }
$$

The beam energy spread also has an equilibrium value (at $\mathrm{J}=0.13)$ of $\delta \mathrm{E}_{\mathrm{rms}}=0.392 \mathrm{MeV}\left(\delta_{\mathrm{rms}}=\right.$ 0.0078).

The longitudinal equilibrium emittance requires a more explicit description of the rf system. The present parameters require an energy recovery rf of $\mathrm{zeV}_{\text {rf }}$ of more than $1.87 \mathrm{MeV}$ per turn or $\mathrm{V}_{\text {rf }}$ $>0.61 \mathrm{MV}(\mathrm{z}=3)$. If we set $\mathrm{V}_{\mathrm{rf}}$ at $0.9 \mathrm{MV}$, we get $\phi_{\mathrm{s}}=47^{\circ}$, and the bunching voltage $\mathrm{V}_{\mathrm{rf}} \sin \phi_{\mathrm{s}}$ is $0.66 \mathrm{MV}$. If the rf wavelength is $26.6 \mathrm{~m}$ and $\mathrm{C}=10 \mathrm{~m} \quad\left(\mathrm{f}_{\mathrm{rf}}=11.28 \mathrm{MHz}, \mathrm{h}=4\right)$ and $\gamma_{\mathrm{t}}=2$, we obtain $\beta_{\mathrm{L}}=8.7 \mathrm{~m}$, using the equation:

$$
\beta_{\mathrm{L}}=\sqrt{\frac{\left\langle\mathrm{z}^{2}\right\rangle}{\left\langle\delta^{2}\right\rangle}}=\sqrt{\frac{\beta^{2} \mathrm{PcC} \lambda_{\mathrm{RF}}\left(\frac{1}{\gamma^{2}}-\frac{1}{\gamma_{\mathrm{T}}^{2}}\right)}{2 \pi \mathrm{zeV} \mathrm{V}_{\mathrm{RF}} \sin \varphi_{\mathrm{S}}}} .
$$

The equilibrium longitudinal emittance is:

$$
\varepsilon_{\mathrm{L}, \mathrm{N}}=\frac{\beta_{\mathrm{L}} \beta \gamma \frac{\mathrm{d} \delta_{\mathrm{rms}}^{2}}{\mathrm{ds}}}{2 \mathrm{~J}_{\mathrm{Z}} \frac{1}{\mathrm{P}} \frac{\mathrm{dP}}{\mathrm{ds}}}
$$

which would obtain $\varepsilon_{\mathrm{L}, \mathrm{N}}=\sim 5.0 \times 10^{-4} \mathrm{~m}$ (per bunch). This would imply an rms bunchlength of $\delta \mathrm{z}=0.07 \mathrm{~m}$, which is fairly short ( $\delta \phi_{\mathrm{rms}}=0.17$ radians), indicating that the longitudinal bunching is relatively strong, and somewhat weaker longitudinal focusing could be used. (However, $\delta_{\text {rms }}$ does not depend upon the bunching factor.)

We summarize some of these parameters in table 1. 
The ionization cooling target $\left({ }^{3} \mathrm{He}\right)$ can probably not be provided in liquid or solid form. Ref. 1 proposes using a gas jet formed into the wedge shape needed for emittance exchange. The gas jet has many challenging design and operation features.

A serious problem that is not completely resolved in ref. 1 is the difficulty of separating the desired production ions $\left({ }^{8} \mathrm{~B}\right)$ from the circulating Li- 6 beam. In ref. 1 , the ions are stopped on a material downstream from the target. However, this stop must have a hole large enough that the circulating Li-6 ions will not intersect. The Li-6 ions emerge from the target with an rms spread of $2^{\circ}$ and we would need an aperture of $>\sim 4 \sigma$ to avoid large losses, and even more room to accommodate the momentum spread (if dispersion is nonzero at the stopper). The B-8 ions are produced in a forward distribution that extends out to only $\sim 14.5^{\circ}$. A significant fraction of these must pass through the beam aperture, and would be lost. An additional kicker to separate the beams may be desirable.

As noted in ref. 1, space charge limitations are a significant problem, particularly in the storage of low-energy high-charge ions. A measure of the strength of space charge forces is the spacecharge tune shift, which is given by the formula:

$$
\delta v=\frac{\mathrm{z}^{2} \mathrm{r}_{\mathrm{p}} \mathrm{N}_{\text {tot }}}{4 \pi \beta \gamma^{2} \mathrm{aB}_{\mathrm{F}} \varepsilon_{\mathrm{N}, \mathrm{rms}}}
$$

For the parameters we are considering $\left(\mathrm{N}_{\mathrm{TOT}}=10^{12}, \mathrm{z}=3, \mathrm{a}=6, \beta=0.094\right.$ and $\left.\varepsilon_{\mathrm{N}, \mathrm{rms}}=\sim 4.35 \times 10^{-5} \mathrm{~m}\right)$, we obtain $\delta v=0.045 / \mathrm{B}_{\mathrm{F}}$, indicating that we need $\mathrm{B}_{\mathrm{F}}$ to be greater than $\sim 0.1$ to obtain $\delta v<0.5$. (At the above parameters, $\mathrm{B}_{\mathrm{F}} \cong 0.17 * 2.5 / 6.28 \cong 0.07$, so somewhat weaker longitudinal focusing is desired and will not be too difficult to obtain.)

\section{Discussion: "Reverse” or "Direct” kinematics?}

The B-8 ions can also be produced by He-3 ions moving through a Li- 6 absorber/target and ionization cooling can be used to obtain multiturn passages of beam through absorbers for cooling. In ref. 1 this configuration would be described as the direct case, while Li-6 ions passing through He-3 absorbers is referred to as "reverse" kinematics.

Since the mass of He-3 is half that of Li-6, the collisions will occur at the same center of mass energy if the kinetic energy of the He-3 is half the reference value of the previous example. $\left(\mathrm{T}_{\mathrm{He}}=\right.$ $12.5 \mathrm{MeV})$. At these parameters $\beta$ is the same as before $(\beta=0.094)$, which means that the partition numbers are approximately the same. For initial comparisons we set $\beta_{\perp}=0.3 \mathrm{~m}$ and $\eta=\Delta_{x}=0.3 \mathrm{~m}$ for both examples and use the same ring circumference for both cases. (Since the magnetic rigidity is a factor of $3 / 4$ less, a strict scaling could allow us to reduce the circumference by that factor, which would be advantageous.)

The optimum energy for storage is not yet fully established. Reference 1 shows cross sections for ion production as a function of energy and assumes that the total cross section is 1 barn, independent of energy. The optimum energy would depend on the ratio of ions produced and accepted to actual total cross section, and this more complete evaluation is needed, and the optimum may differ for direct from reverse kinematics.

Parameters of this direct-kinematics example are included in Table 1. Because the product $\mathrm{L}_{\mathrm{R}} \mathrm{dE} / \mathrm{ds}$ is a factor of $\sim$ two higher for ions passing through $\mathrm{Li}$ than $\mathrm{He}$, the normalized 
equilibrium emittance is larger by that factor, which would make beam sizes and angles a factor of $\sim(2)^{1 / 2}$ larger. That is a potential disadvantage of the direct kinematics.

A potential advantage of the direct kinematics is that space charge is naturally less by a factor of 2.25 from the $\mathrm{z}^{2}$ factor and by another factor of 2 from the larger natural $\varepsilon_{\mathrm{N}}$. This means that roughly $4.5 \times$ as many ions could be stored, which would allow us to increase the storage time (for the same final number of ${ }^{8} \mathrm{~B}$ ) by that factor, which would then allow us to reduce the absorber thickness by that factor. (The reduced thickness would be used to reduce the possibility of ${ }^{8} \mathrm{~B}$ ions being stopped in the absorber, compensating for the fact that the ${ }^{8} \mathrm{~B}$ ions are produced at lower energies and a correspondingly higher stopping probability.)

A potential difficulty is that the B-8 ions are produced at smaller energies and velocities than the circulating He-3 beam, while in reverse kinematics the B-8 and Li-6 ions are similar in energy and velocity. This increases the chance that the B-8 ions may be trapped in the absorbers; however, it may also make it easier to separate the circulating beam from the produced nuclei.

Thus, a very important potential advantage of the direct kinematics is that the ${ }^{8} \mathrm{~B}$ ions are produced at energies and velocities much less than that of the circulating He-3 beam. This could make it much easier to separate them. The separation in speed could make it much easier to use an rf separator or pulsed kicker to deflect the ${ }^{8} \mathrm{~B}$ away from the circulating beam.

The repeated passages of the beam through the target deposit a large amount of energy in the thin absorber ( $\sim 1 \mathrm{MW}$ for the reverse kinematics case, but $\sim 0.5 \mathrm{MW}$ for the direct kinematics). It is unlikely that a solid could survive the heating, so a ${ }^{3} \mathrm{He}$ absorber would be inserted as a gas jet. A ${ }^{6} \mathrm{Li}$ absorber could be introduced as a liquid "waterfall" or jet, similar to those designed for fusion reactors, but it would be a relatively thin jet ( $\sim 75 \mu$ thick). Both target styles would pose interesting practical difficulties.

${ }^{3} \mathrm{He}$ is more difficult to obtain and to handle than ${ }^{6} \mathrm{Li}$ and, without very efficient recirculation, the jet-source will use relatively large amounts of material. This may be an important advantage for the direct production. Also the ${ }^{3} \mathrm{He}$ gas will diffuse throughout the accelerator system much more easily than ${ }^{6} \mathrm{Li}$ liquid (which would condense outside the hot target). The probably inferior vacuum will add to particle loss and scattering, and electron-ion instability.

The practical difficulties associated with the reverse kinematics may be greater than in the direct case, and the direct approach (He-3 beam and Li-6 target) may be preferable. Future studies should consider both options.

\section{Further Discussion}

The above discussion focuses on the use of a cooled ion beam to enhance the production and acceptance of ${ }^{8} \mathrm{~B}$ for a beta-beam source for the production of neutrinos. Similar considerations would apply to ${ }^{8} \mathrm{Li}$ production from the reaction ${ }^{7} \mathrm{Li}+{ }^{2} \mathrm{H} \rightarrow{ }^{8} \mathrm{Li}+{ }^{1} \mathrm{H}$ for an antineutrino source. This antineutrino source is relatively easy because the cross-section for ${ }^{8} \mathrm{Li}$ production is an order of magnitude larger than the ${ }^{8} \mathrm{~B}$ production, and the ${ }^{2} \mathrm{H}$ source is easier to obtain. We will not describe this scenario in more detail in the present paper. As in the above case, direct or reverse kinematics can be chosen.

Production of ${ }^{6} \mathrm{He}$ for a beta-beam antineutrino source is also being considered and a relatively intense source can be obtained without the multiturn target and ion cooling scenario of the present 
approach, since the noble-gas status of the ${ }^{6} \mathrm{He}$ makes it easier to separate target from product ion. The difficulty is in obtaining a good neutrino source; the nearest noble gas neutrino source is ${ }^{18} \mathrm{Ne}$, and it is much more difficult to produce and collect. The presently discussed ${ }^{8} \mathrm{~B}$ source could be more effective.

We have freely used the rms ionization cooling equations as accurate in a regime where the longitudinal heating nearly cancels the transverse cooling, and nuclear effects are ignored. (These and other effects could cancel out the residual cooling effect and make the method impractical.) We note that Y. Mori and collaborators are constructing a similar device (an FFAG ring) in which protons are recirculated through a Be foil target for multiturn neutron production, and are relying on beam cooling dynamics to extend the proton beam lifetime.[8] Their experiences will enable some direct evaluation of the practicality of ionization cooling in this type of device.

In summary, the use of stored ions to produce radioactive ions within a configuration in which the thin production target is also used to cool the stored ions to enable many-turn production collisions is a promising possibility that could enable production of an intense Boron- 8 source for electron-neutrino beams. Our analysis indicates that the scenario must be modified from that of ref. 1 to enable coupling of $\mathrm{x}, \mathrm{y}$, and $\mathrm{z}$ to obtain 3-D cooling, and that the equilibrium emittances are somewhat larger than those estimated in ref. 1. Even though the enlarged emittances require larger ring apertures, they also reduce the space charge difficulty noted in ref. 1 to manageable levels. A direct kinematics scenario with a He-3 beam and a $\mathrm{Li}$ absorber is possible. Much further design, analysis, and optimization is needed to find a complete scenario. 
Figure 1: The longitudinal partition number, $\mathrm{J}_{\mathrm{z}}$, and the sum of $\mathrm{x}, \mathrm{y}, \mathrm{z}$ partition numbers, $\Sigma_{\mathrm{J}}=2+$ $\mathrm{J}_{\mathrm{z}}$ as a function of muon momentum $\mathrm{m}_{\mu} \beta \gamma \mathrm{c}$. $\Sigma_{\mathrm{J}}$ varies from $\sim 0.36$ at $\mathrm{P}_{\mu}=20 \mathrm{MeV} / \mathrm{c}$ to slightly more than 2 for $\mathrm{P}_{\mu}>350 \mathrm{MeV} / \mathrm{c}$. This particular graph is for hydrogen absorbers (with $\delta=0$ ); there is only a weak dependence on absorber material. The parameters depend only on $\beta=\mathrm{v} / \mathrm{c}$ of the incident particle. (Ions have the same $\Sigma_{\mathrm{J}}$ as muons with $\left.\mathrm{P}_{\mu}=\left(\mathrm{m}_{\mu} / \mathrm{am}_{\mathrm{N}}\right) \mathrm{P}_{\mathrm{a} .}\right)(\beta=0.094$ corresponds to a muon momentum of $\sim 10 \mathrm{MeV} / \mathrm{c}$, where $\Sigma \mathrm{J}_{\mathrm{i}}$ is $\sim 0.4$.)

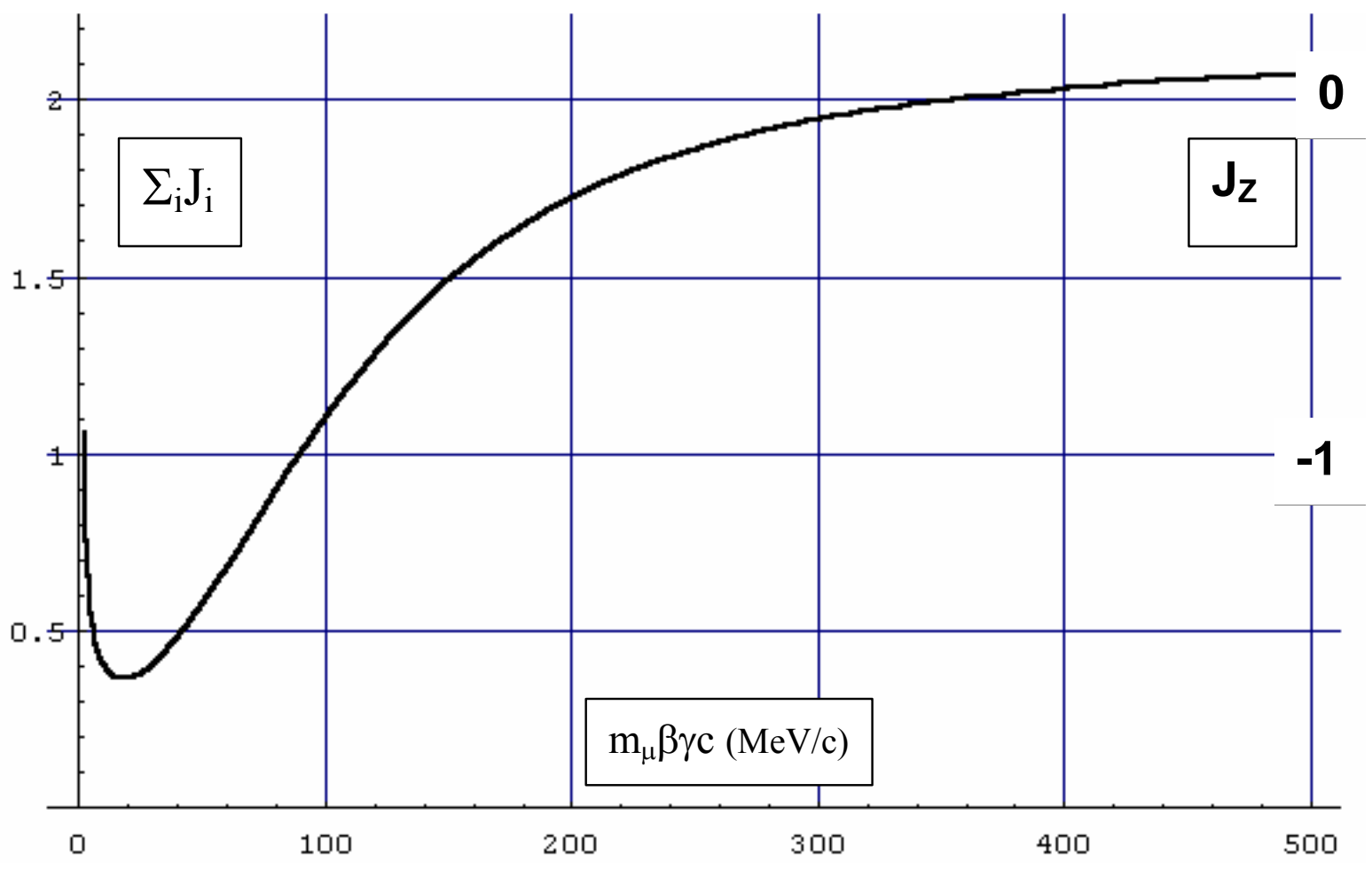


Table 1: Low-Energy Ion Cooling Examples

\begin{tabular}{|c|c|c|c|}
\hline \multicolumn{4}{|c|}{ Parameters for Ion Cooling for production of B-8 } \\
\hline Parameter & Symbol & Reverse Dynamics & Direct Scenario \\
\hline Beam & & ${ }^{6} \mathrm{Li}$ & ${ }^{3} \mathrm{He}$ \\
\hline Absorber & & ${ }^{3} \mathrm{He}$ & ${ }^{6} \mathrm{Li}$ \\
\hline Momentum & $\mathrm{P}$ & $530 \mathrm{MeV} / \mathrm{c}$ & $265 \mathrm{MeV} / \mathrm{c}$ \\
\hline Kinetic energy & $\mathrm{T}_{\mathrm{a}}$ & 25 & $12.5 \mathrm{MeV}$ \\
\hline Speed & $\beta=v / c$ & 0.094 & 0.094 \\
\hline Absorber density (reference) & $\rho_{\text {ref }}($ liquid or solid $)$ & 0.09375 & 0.46 \\
\hline Energy loss & $\mathrm{dE} / \mathrm{ds}$ & $110.6 \mathrm{MeV} / \mathrm{cm}$ & $170.4 \mathrm{MeV} / \mathrm{cm}$ \\
\hline Radiation Length & $\mathrm{L}_{\mathrm{R}}$ & $756 \mathrm{~cm}$ & $155 \mathrm{~cm}$ \\
\hline Betatron functions at absorber & $\beta_{\perp}, \eta$ & $0.3 \mathrm{~m}, 0.3 \mathrm{~m}$ & $0.3 \mathrm{~m}, 0.3 \mathrm{~m}$ \\
\hline Rms angle & $\delta \theta_{\mathrm{rms}}\left(\beta_{\mathrm{t}}=0.3 \mathrm{~m}\right)$ & $2.25 \mathrm{~K}_{\mathrm{s}}^{\circ}$ & $3.8 \mathrm{~K}_{\mathrm{s}}^{\circ}$ \\
\hline Rms beam size & $\sigma_{t}\left(\right.$ at $\left.\beta_{t}=1 \mathrm{~m}\right)$ & $2.15 \mathrm{~K}_{\mathrm{s}} \mathrm{cm}$ & $3.6 \mathrm{~K}_{\mathrm{s}} \mathrm{cm}$ \\
\hline $\begin{array}{l}\text { Absorber thickness (3000 turn } \\
\text { lifetime) }\end{array}$ & $\lambda_{\text {abs }}$ & $0.018 \mathrm{~cm}$ & $0.00725 \mathrm{~cm}$ \\
\hline Characteristic Cooling Length & $(\mathrm{dP} / \mathrm{ds} / \mathrm{P})^{-1}$ & $0.45 \mathrm{~cm}$ & $0.147 \mathrm{~cm}$ \\
\hline Multiple Scattering & $\mathrm{d}\left(\theta^{2}\right) / \mathrm{ds}$ & $8.84 \times 10^{-4} \mathrm{~K}_{\mathrm{s}}^{2} / \mathrm{cm}$ & $0.0078 \mathrm{~K}_{\mathrm{s}}^{2} / \mathrm{cm}$ \\
\hline Straggling & $\mathrm{d}\left(\delta \mathrm{E}^{2}\right) / \mathrm{ds}$ & $0.0886 \mathrm{MeV}^{2} / \mathrm{cm}$ & $0.143 \mathrm{MeV}^{2} / \mathrm{cm}$ \\
\hline Sum of partition numbers. & $\sum \mathrm{J}_{\mathrm{i}}$ & 0.4 & 0.4 \\
\hline Eq. transverse emittance & $\varepsilon_{\mathrm{T}, \mathrm{N}, \mathrm{rms}}$ & $4.35 \times 10^{-5} \mathrm{~K}_{\mathrm{s}}^{2} \mathrm{~m}$ & $0.000123 \mathrm{~K}_{\mathrm{s}}^{2} \mathrm{~m}$ \\
\hline Equilibrium $\delta \mathrm{P} / \mathrm{P}\left(\mathrm{J}_{\mathrm{z}}=0.13\right)$ & $\delta_{\mathrm{rms}}$ & 0.0078 & 0.0115 \\
\hline Production energy & $\mathrm{E}_{\mathrm{B}-8}$ & 8.3 to $21.5 \mathrm{MeV}$ & 0.93 to $8.3 \mathrm{MeV}$ \\
\hline Production speed & $\beta_{\mathrm{B}-8}$ & 0.047 to 0.078 & 0.016 to 0.047 \\
\hline Maximum production angle & $\theta_{\max }$ & $14^{\circ}$ & $30^{\circ}$ \\
\hline
\end{tabular}

\section{References}

[1] C. Rubbia, A. Ferrari, Y. Kadi, V. Vlachoudis, Nucl. Inst. and Meth. A 568, 475 (2006).

[2] P. Zucchelli, Physics Letters B 532, p. 166 (2002).

[3] D. Neuffer, Nucl. Inst. and Meth. A532, p.26 (2004).

[4] W. M. Yao et al. (Particle Data Group), Journal of Physics G 33, 1 (2006)

[5] G. R. Lynch and O. I. Dahl, Nucl. Inst. and Meth. B58, 6 (1991).

[6] C. X. Wang and K. J. Kim, MuCOOL Note 240 (2002).

[7] R. Talman, “A Proposed Moebius Accelerator”, Phys. Rev. Lett. 74, p. 1592 (1995)

[8] Y. Mori, Nucl. Inst. and Meth. A562, 591 (2006) 